\title{
Multidirectional Vector Excision Leads to Better Outcomes than Traditional Elliptical Excision of Facial Congenital Melanocytic Nevus
}

\author{
Seung Il Oh, Yoon Ho Lee \\ Department of Plastic and Reconstructive Surgery, Seoul National University School of Medicine, Seoul, Korea
}

Background The elliptical excision is the standard method of removing benign skin lesions, such as congenital melanocytic nevi. This technique allows for primary closure, with little to no dog-ear deformity, but may sacrifice normal tissue adjacent to the lesion, resulting in scars which are unnecessarily long. This study was designed to compare the predicted results of elliptical excision with those resulting from our excision technique.

Methods Eighty-two patients with congenital melanocytic nevus on the face were prospec tively studied. Each lesion was examined and an optimal ellipse was designed and marked on the skin. After an incision on one side of the nevus margin, subcutaneous undermining was performed in the appropriate direction. The skin flap was pulled up and approximated along several vectors to minimize the occurrence of dog-ear deformity.

Results Overall, the final wound length was $21.1 \%$ shorter than that achieved by elliptical excision. Only $8.5 \%$ of the patients required dog-ear repair. There was no significant distortion of critical facial structures. All of the scars were deemed aesthetically acceptable based on their Patient and Observer Scar Assessment Scale scores.

Conclusions When compared to elliptical excision, our technique appears to minimize dogear deformity and decrease the final wound length. This technique should be considered an alternative method for excision of facial nevi.

Keywords Nevus / Cicatrix / Wound closure technique
Correspondence: Yoon Ho Lee Department of Plastic and Reconstructive Surgery, Seoul National University School of Medicine, 101 Daehak-ro, Jongno-gu, Seoul 110-744, Korea

Tel: +82-2-2072-2374

Fax: +82-2-3675-7792

E-mail:lyh2374@snu.ac.kr

Received: 7 Mar 2013 • Revised: 1 Jul 2013 • Accepted: 4 Jul 2013

pISSN: 2234-6163 • elSSN: 2234-6171 • http://dx.doi.org/10.5999/aps.2013.40.5.570 • Arch Plast Surg 2013;40:570-574

\section{INTRODUCTION}

Congenital melanocytic nevi (CMN) of the face are common malformations that are very problematic for patients. The optimal management of CMN has been a longstanding surgical challenge, due to the need for a complete excision of the lesion and the problem of aesthetic disfigurement from scarring [1-4].

The traditional method for nevus excision is elliptical design. This pattern allows primary closure without dog ear formation.
Usually, the recommended length-to-width ratio is between 3.0 and $4.5[5,6]$, but this results in a longer scar than the primary lesion. The surgeon usually sacrifices a significant amount of normal skin, as attempts to excise less skin result in dog ear formation.

To minimize tension for wound approximation, wider subcutaneous undermining is required. After wound closure, however, the distortion of important normal facial structure is possible. Planning the width of the excision is not easy, because the tissue 
tension after wider undermining and the distortion of normal structures are usually not equal to those predicted preoperatively. Unfortunately, changing the plan to serial excision because of excessive tension is generally inappropriate after the removal of a total lesion.

In this study, we carefully compared the outcomes of the predicted results of planned elliptical designs with those achieved after excision with our alternative technique using one side incision and multidirectional vector wound closure.

\section{METHODS}

A total of 82 patients with CMN were treated with this technique in our institution during the period from 2000 to 2012 . 34 were males, and 48 were females. Their age ranged from 6 months to 31 years. Each lesion was examined and an excision margin along the nevus border was drawn on the face. The potential scar length with an elliptical excision method was calculated as 3 times the planned excision width. In practice, we incised only one side margin of the nevus-the side which was closer to a critical structure such as an eyebrow, nose, eyelid, or lip (Figs. 1, 2). After subcutaneous undermining beneath the lesion, extensive dissection of normal tissue was necessary for approximation following nevus removal. The direction of undermining should be in the opposite direction of critical structures to avoid the distortion of normal facial anatomy. Once adequate undermining was performed, the skin flap including the nevus was pulled over the normal skin with skin hooks. The optimal vectors of skin hook traction were not even because the factors that affect tension and dog-ear deformity-the elastic properties of skin, wound geometry, and surface contour-vary with the location. Ideal vectors are those that cause minimal dog-ear deformity and produce reasonable tension for wound approximation. After carefully deciding on the vectors of traction, the flap was divided in several pieces with slit incisions made by a scalpel at a right angle to the defect margin. If we predict excessive tension from the excision of the entire nevus, the plan should be changed to a partial excision for better postoperative results and slit incisions should be limited to a proper portion of the total width. Several key sutures were then placed at the apices of the notch, followed by the confirmation of normal structure distortions and dog-ear deformity. If a distortion of a facial subunit is definite, the flap to be removed should be designed to be smaller. Despite choosing an ideal vector to minimize dog-ear deformity, sometimes some minor dog-ear formation cannot be avoided. In such cases, mounds of excess skin were removed via a standard dogear correction technique. If the key sutures are placed with minimal distortion and unnoticeable dog-ear deformities, the remaining nevus is delicately removed with curved scissors or a scalpel. The wound is closed in two layers using 5.0 Vicryl and 6.0 Prolene. The sutured wound length was measured and compared with the potential wound length of an elliptical excision (Fig. 3). All of the patients were evaluated in outpatient clinics with the Patient and Observer Scar Assessment Scale (POSAS) 3 months postoperatively [7].

\section{RESULTS}

Twenty-seven nevus excisions were conducted on the forehead, 38 on the cheek, 11 on the chin, and 6 on the nose (Figs. 4, 5). The size of the nevi varied between $8 \mathrm{~mm}$ and $52 \mathrm{~mm}$ (mean, $17.5 \mathrm{~mm})$. Seventy-five of the wounds $(91.5 \%)$ required no dog-ear repair, 5 wounds $(6.1 \%)$ required 1 dog-ear repair, and 2 wounds (2.4\%) required 2 dog-ear repairs. All of the sutured wounds were shorter than the potential wounds predicted from an elliptical design, and the mean length of sutured wounds was $14 \%$ shorter than the expected elliptical wound length (Table 1). There was no significant distortion of normal structures. Complications (dehiscence, hematoma, infection, and skin necrosis) were not observed during postsurgical observation (range, 6-24 months; mean, 10.2 months). All of the scars were deemed aesthetically reasonable based on their POSAS scores.

\section{DISCUSSION}

The most common excision skin pattern for nevi is a fusiform or

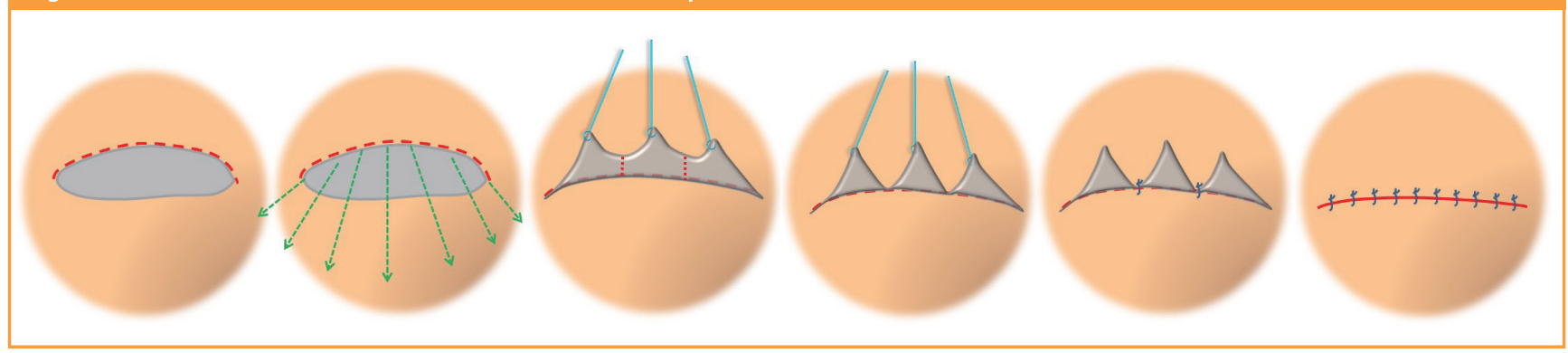


Fig. 2. Congenital melanocytic nevus on the left cheek

(A) Immediately after an incision on one side, nearer critical facial subunits, the nose and lip. (B) Undermining toward the direction opposite the critical subunit to minimize distortion after wound closure. (C) Traction of the skin flap toward the ideal vector and division in pieces. (D) Confirmation of no noticeable dog ear and reasonable tension after placing a temporary suture. (E) Final wound.

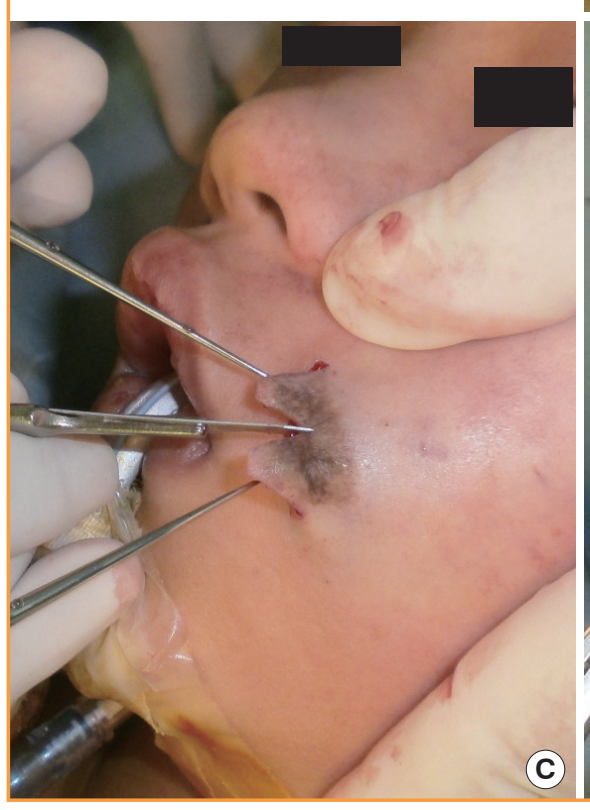

(C)
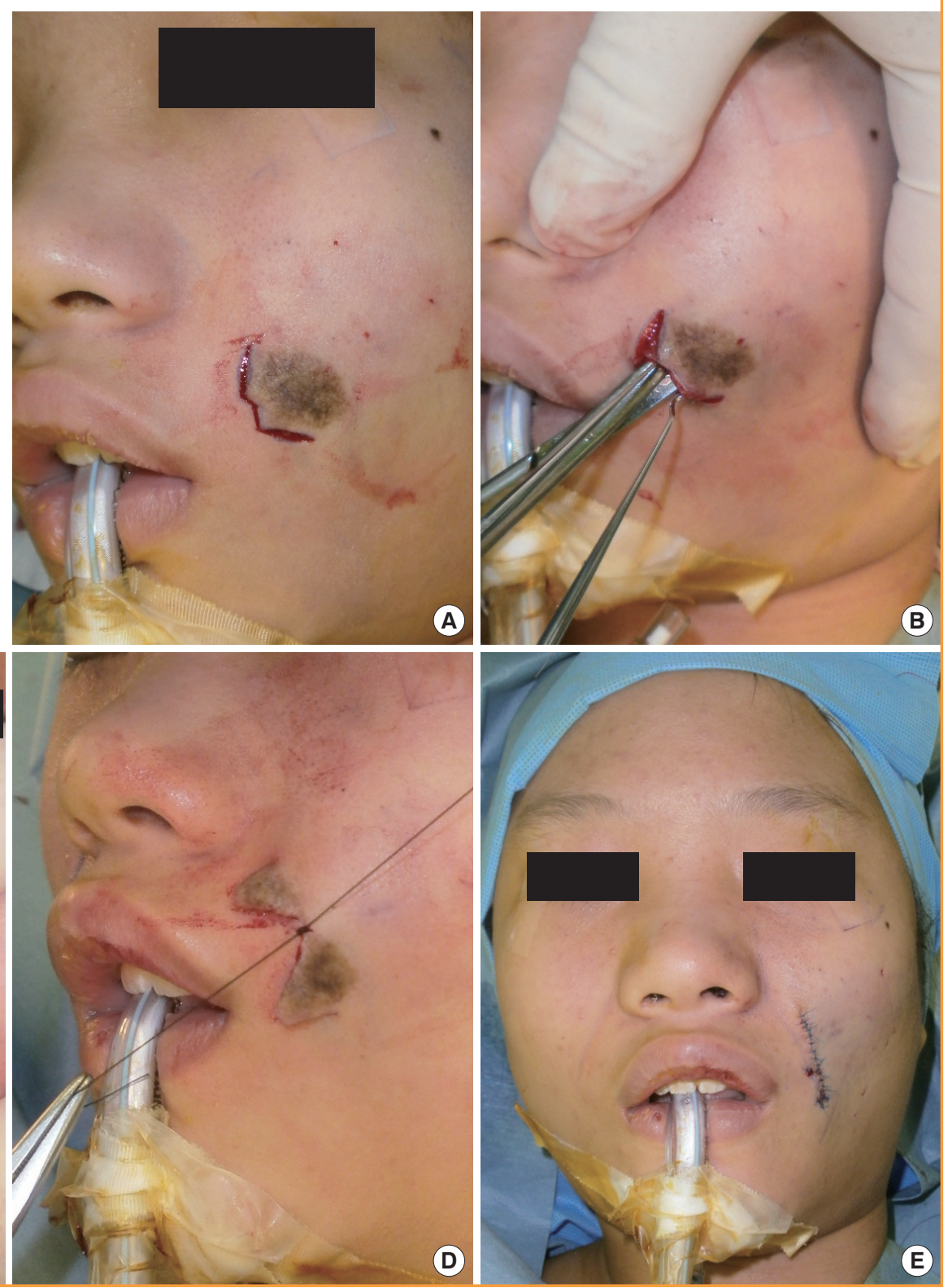

elliptical shape. The usefulness and efficacy of this pattern have been well described in the literature $[5,6]$. This design allows for the primary closure of the defect without dog-ear deformities. However, there are some significant disadvantages to this technique. One major disadvantage is that the length of the final wound becomes longer than the primary lesion. Commonly, for this design, a length-to-width ratio between 3.0 and 4.5 is recommended. Another disadvantage is the wasting of an excessive amount of healthy tissue to avoid dog-ear deformities. This can create significant aesthetic problems when the lesion is near a critical facial structure, such as an eyebrow, nose, eyelid, or lip. Lastly, the design of this type of incision poorly compensates for the variability in regional tissue laxity.
A dog-ear deformity is a protrusion of soft tissue that forms during wound approximation. It occurs most commonly when a simple elliptical excision is performed with a long axis that is too short compared with the width. The dog ear is more noticeable than the fine linear scar of a well-closed wound. Therefore, elliptical excision without dog ears, even at the expense of lengthening the wound, has traditionally been the most important principle in wound closure [8]. However, this may waste an excessive amount of healthy tissue, and can also result in poor outcomes in both the wound length and direction, as the surgeon plans the design before performing the excision of a lesion. The literature reports considerable variation among planned excisions, wounds, and defect sizes that can be affected by the lesion's loca- 


\section{Fig. 3. Schematic demonstration of measurement of scar length difference}

$a$, final wound length of our method; $b$, potential wound length of elliptical design.

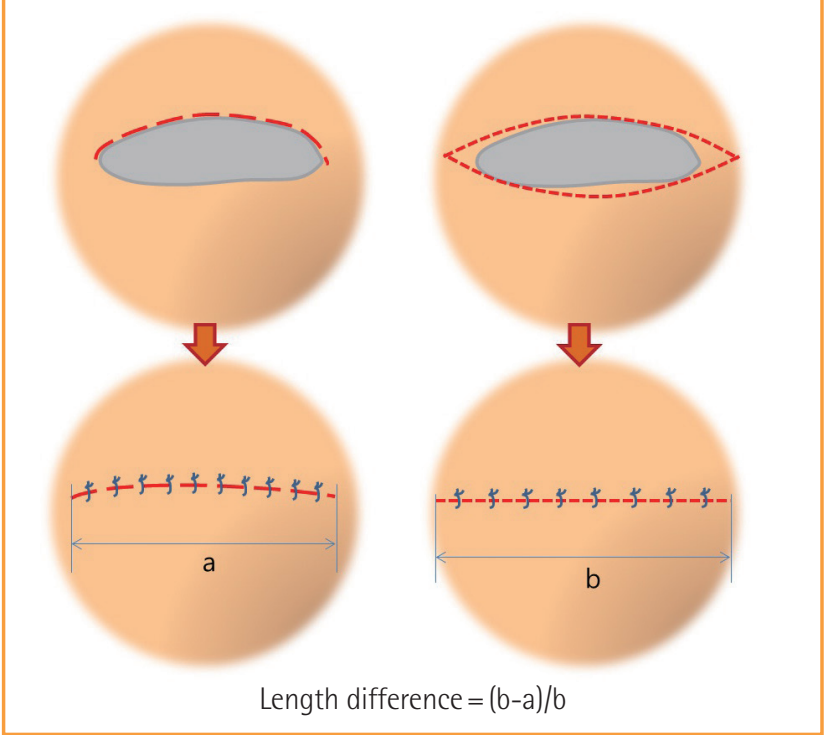

\section{Fig. 4. Congenital melanocytic nevus on the left cheek}

(A) Preoperative photograph $(21 \mathrm{~mm} \times 15 \mathrm{~mm})$. (B) Postoperative photograph on month 3 with a linear scar of $38 \mathrm{~mm}$, showing an unnoticeable dog-ear deformity and no distortion of the facial unit.
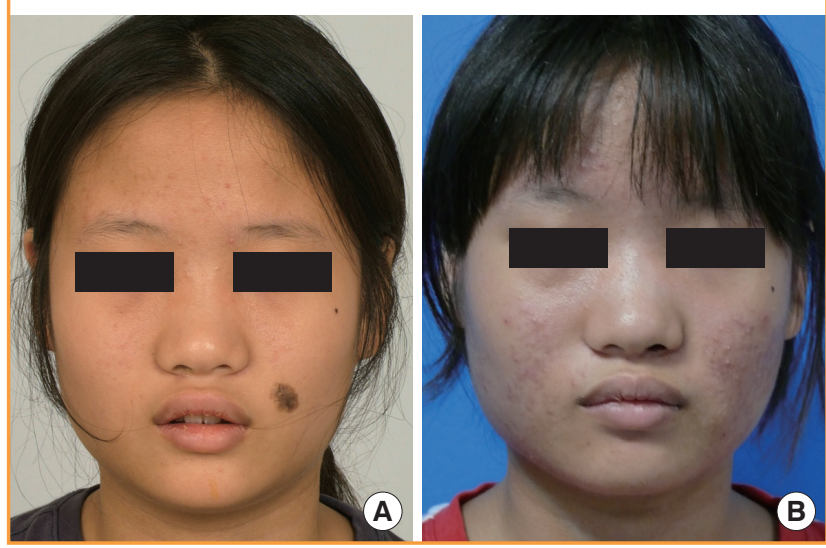

tion and shape [9]. In addition, several factors influence dog-ear formation including tissue dynamics, wound geometry, surface contour, and surgical technique [10]. Therefore, it is not reasonable to commit to sacrificing healthy tissue prior to measuring the exact shape and size of the defect that will appear after nevus removal. The optimal tissue traction vectors to minimize dogear formation can be identified after all possible directions have been assessed. In this manner, we can use adjacent skin with the maximum stretchability.

For wound approximation, usually wide undermining is performed all around the defect. This can help to reduce tension for wound closure, but often brings out some distortion of a nearby

\section{Fig. 5. Congenital melanocytic nevus on the right cheek}

(A) Preoperative photograph $(37 \mathrm{~mm} \times 21 \mathrm{~mm})$. (B) Photograph at 3 months postoperatively with a linear scar of $51 \mathrm{~mm}$, showing no dog-ear deformity and a reasonable scar length.
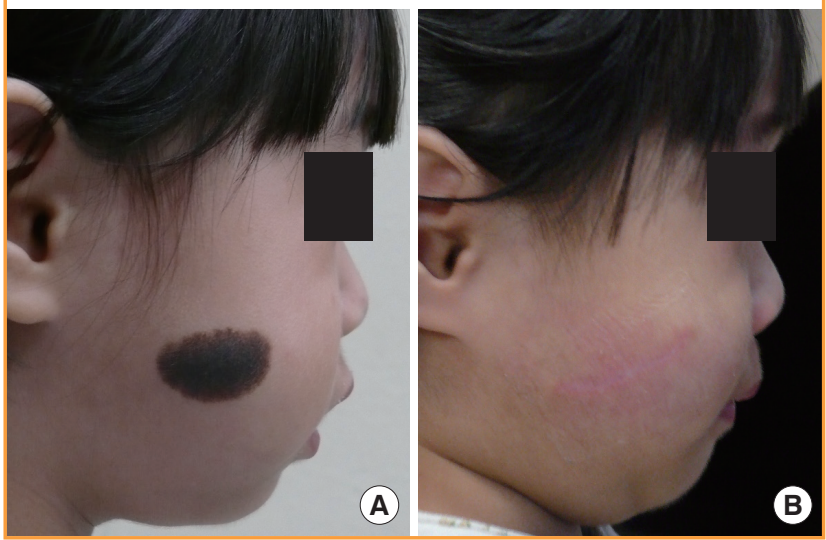

Table 1. Comparison of planned elliptical excision and actual wound length by site

\begin{tabular}{|lcccl|}
\hline Site & Cases & $\begin{array}{c}\text { Average } \\
\text { length of } \\
\text { primary } \\
\text { lesion (mm) }\end{array}$ & $\begin{array}{c}\text { Length } \\
\text { difference } \\
\mathbf{( \% )}\end{array}$ & $\begin{array}{c}\text { Dog } \\
\text { ears }\end{array}$ \\
\hline Forehead & 27 & 15.4 & 12.8 & 0 \\
Cheek & 38 & 21.4 & 15.4 & 4 \\
Chin & 11 & 13.5 & 14.3 & 2 \\
Nose & 6 & 9.6 & 10.0 & 1 \\
Total & 82 & 17.5 & 14.0 & $7(8.5 \%)$ \\
\hline
\end{tabular}

critical facial structure such as an eyebrow, eyelid, nose, or lip. In our technique, we perform subcutaneous undermining for tension reduction not in all directions but toward directions opposite of critical structures. This way, we can minimize the distortion of facial structures even though the area for undermining is usually wider than in conventional methods.

Excessive tension in wound closure is a negative factor that can contribute to scar widening or depression. In conventional methods, tension after a nevus excision and primary closure is predicted before operation by examining the elasticity and thickness of the surrounding normal tissue. However, this way, the tension of the sutured wound does not always correspond with that predicted preoperatively. Unfortunately, despite finding out that the tension is excessive, it is not easy to change the plan of a partial excision after the removal of a total lesion. The advantage of our method is that if the tension for approximation is more excessive than what was predicted preoperatively, the width of the excision can be reduced intraoperatively to allow a reasonable level of tension,. Based on our experience, nevi of sizes less than or equal to $3 \mathrm{~cm}$ could be successfully excised with this technique. Nevi larger than $3 \mathrm{~cm}$, on the other hand, 
may require other methods to avoid dog-ear deformity or excessive tension.

The limitations of our study include the lack of validation of our results by a randomized control trial. Although the methods described in our study have been applied successfully to our patients, a larger study with more surgeons may help confirm our conclusions and provide new perspectives.

We believe that, for the excision and closure of facial nevi, this technique should be considered a generally superior method over conventional elliptical excision. Our results also show that compared with elliptical excision, the mean length of the sutured wound was $14 \%$ shorter. In addition, this technique has advantages with respect to unnoticeable dog ears, minimal distortion of critical structures, and intraoperative adjustability of the excision width.

\section{REFERENCES}

1. Alikhan A, Ibrahimi OA, Eisen DB. Congenital melanocytic nevi: where are we now? Part I. Clinical presentation, epidemiology, pathogenesis, histology, malignant transformation, and neurocutaneous melanosis. J Am Acad Dermatol 2012;67:495. e1-17.

2. Kim SW, Kim JH, Lee Vh, et al. Surgical treatment of con- genital melanocytic nevus in the face of the children. J Korean Soc Plast Reconstr Surg 1998;25:1121-6.

3. Jo SH, Kim JW, Jung JH, et al. Serial Reconstruction Considerating the Aesthetic Unit on Congenital Giant Nevus in Periorbital Area. J Korean Soc Plast Reconstr Surg 2010;37: 465-8.

4. Arneja JS, Gosain AK. Giant congenital melanocytic nevi. Plast Reconstr Surg 2007; 120:26e-40e.

5. Bennett RG. Fundamentals of cutaneous surgery. St. Louis: Mosby; 1988.

6. DeHoff PH, Key JE. Application of the finite element analysis to determine forces and stresses in wound closing. J Biomech 1981;14:549-54.

7. Draaijers LJ, Tempelman FR, Botman YA, et al. The patient and observer scar assessment scale: a reliable and feasible tool for scar evaluation. Plast Reconstr Surg 2004;113:1960-5.

8. Seo SH, Son SW, Kim IH. Round excisions lead to shorter scars and better scar positioning than traditional elliptical excisions. Dermatology 2008;217:276-80.

9. Hudson-Peacock MJ, Matthews JN, Lawrence CM. Relation between size of skin excision, wound, and specimen. J Am Acad Dermatol 1995;32:1010-5.

10. Weisberg NK, Nehal KS, Zide BM. Dog-ears: a review. Dermatol Surg 2000;26:363-70. 